\title{
Auditory Middle Latency Response in Young Adult Females Perceiving Tinnitus During a Brief Period of Silence
}

\section{Marwa F Abdrabbou and Denise A Tucker*}

Department of Communication Sciences and Disorders, University of North Carolina

at Greensboro, USA

*Corresponding Author: Denise A Tucker, Department of Communication Sciences and Disorders, University of North Carolina at Greensboro, USA.
Received: September 30, 2021

Published: November 29, 2021

(C) All rights are reserved by Marwa F

Abdrabbou and Denise A Tucker.

\section{Abstract}

Background: Brief silence exposure plays an important role in the temporary emergence of tinnitus in normal hearing subjects with no chronic tinnitus complaints. Several studies reported a high prevalence of tinnitus perception during silence in their cohort. However, none of these studies examined the effect of silence and tinnitus perception on the central auditory functions to determine the role of silence in these perceptions.

Purpose: This study examines the emergence of tinnitus during a brief period of silence exposure without directed auditory attention and its impact on neural activities using auditory middle latency response measurements.

Research Design: Cross-sectional study.

Study Sample: Sixty normal hearing adult females (18-40 years) with no prior tinnitus complaints participated in this study.

Data Collection and Analysis: Auditory middle latency response Na/Pa latency and amplitude were measured before and after ten minutes of silence exposure. Participants completed a brief questionnaire documenting the emergence of tinnitus perceptions during silence. Repeated-measures ANOVA was used to determine clinical significance.

Results: 55\% of the participants reported temporary tinnitus perception during silence without directed auditory attention. Results revealed larger auditory middle latency response $\mathrm{Na} / \mathrm{Pa}$ amplitude in tinnitus-perceiving subjects in both pre-silence and postsilence recordings.

Conclusion: Tinnitus perception may emerge in normal hearing females with no prior tinnitus complaints during a brief silence exposure in the absence of directed auditory attention. These temporary perceptions were associated with increased neural activities as reflected in larger auditory middle latency response $\mathrm{Na} / \mathrm{Pa}$ amplitudes in tinnitus perceiving subjects in both pre-silence and post-silence recordings, indicating that silence was a facilitating factor for these tinnitus perceptions.

Keywords: Tinnitus; Silence; Tinnitus Perception During Silence; Auditory Deprivation; Auditory Middle Latency Response

\section{Abbreviations}

CANS: Central Auditory Nervous System; AEPs: Auditory Evoked Potentials; AMLR: Auditory Middle Latency Response

\section{Introduction}

Tinnitus perceptions are phantom sensations of hearing sounds in the absence of an actual external physical auditory stimulus [1]. Tinnitus can occur as either subjective or objective perceptions. 
Objective tinnitus are caused by real sounds generated inside the body reaching the ears through body tissues conduction, whereas subjective tinnitus is the perception of meaningless sounds that are not associated with any physical sound source [2-5]. Subjective tinnitus can be a transient experience that lasts a few seconds to hours or a chronic disorder when these perceptions are sustained over 3 months [6-9]. Transient tinnitus experiences are typically benign (associated with reversible conditions as listening to loud music) and are especially perceived in very quiet environments $[2,6]$, whereas chronic tinnitus perception is a disorder that can negatively impact the quality of life of those affected [9].

To date, the underlying central neural mechanism of tinnitus is still under investigation. Auditory deprivation associated with various degrees of hearing loss has been proposed to play an important role in cascading a series of neural changes along the Central Auditory Nervous System (CANS) through either altering the balance of inhibition and excitation [6,10] or activating neural plasticity [6], resulting in tinnitus perception. However, auditory deprivation can be provoked in the absence of hearing loss by either using earplugs or sitting in silence inside a sound booth for a few minutes resulting in temporary tinnitus perceptions through the same mechanisms $[10,11]$.

Silence has been widely accepted as an exaggerating factor for tinnitus perception in subjects with tinnitus, hence one of the successful tinnitus management strategies is sound therapy [12-14]. The main purpose of these therapies is to mask tinnitus perceptions and decrease the negative reaction to tinnitus [15]. Additionally, several studies have demonstrated that silence plays a role in the emergence of temporary tinnitus perceptions in healthy normal hearing adults with no prior tinnitus complaints [16-19]. These studies have reported a high prevalence of temporary tinnitus perceptions in their cohort within the first five minutes of silence. These studies examined the prevalence and epidemiology of these perceptions as the effect of gender and race ${ }^{19}$, directed auditory attention and expectation [18], and auditory suggestion [16].

There are two possible explanations for transient tinnitus perception that emerges during silence in the previous studies. The first explanation is that silence exposure might result in the perception of neural activities that have been previously masked by the enriched environment. In such a case silence will not have an impact on CANS activities when measured before and after silence exposure. The second explanation is silence exposure will increase gain within the auditory pathways resulting in tinnitus perception. In this case, silence will have an impact on CANS activities in which post-silence measures will be enhanced compared to presilence measures. Hence, examining CANS activities using Auditory Evoked Potentials (AEPs) before and after a brief period of silence could further our understanding of this phenomenon.

Since AEPs reflect the synchronous discharge of neuronal fibers within the auditory pathway, AEPs recording could potentially be a useful tool to monitor any changes in CANS activity associated with tinnitus perception during silence. Specifically, the Auditory Middle Latency Response (AMLR), which is a non-invasive assessment that can evaluate the integrity of the central auditory system up to the level of the primary auditory cortex [20].

\section{Objectives}

Brief silence exposure has been reported to play a significant role in the emergence of transient tinnitus perceptions in subjects with no prior tinnitus complaints [16-19]. However, none of these previous studies examined the CANS activities associated with tinnitus perception during silence to determine the role of silence in these perceptions. The main purpose of this study is to examine the CANS activities using AMLR measures before and after silence exposure in normal hearing subjects and to report the characteristics of tinnitus perceptions in terms of tinnitus onset, location, and types of sounds perceived by the study participants while attention is directed away from the auditory system. The results of this study will help clarify the role of silence in these perceptions, whether the silence is the provoking factor of the neural changes or just decrease the contrast of the background noise making already exciting enhanced neural activities more perceivable.

\section{Materials and Methods}

\section{Participants}

Sixty normal hearing adult females (18-40 years) participated in this study to control for the effect of age and gender on the AEPs measurements [20]. Eligibility criteria for participation were normal hearing sensitivity (air conduction thresholds $<25 \mathrm{~dB}$ from 250 to $8,000 \mathrm{~Hz}$ ); normal tympanogram (peaks within -100 and +100 dapa); no history of chronic tinnitus; and no history of head trauma, neurologic disease, or ear surgery. Informed consent was obtained prior to any measurements being performed. Institu- 
tional Review Board (IRB \# 18-0096) approved all research procedures for the study. A priori power analysis was conducted using $\mathrm{G}^{*}$ Power with a small to medium effect size $(\mathrm{d}=1.04$ to 0.2$)$, and an alpha $=0.05$ for repeated measure ANOVA measures to determine the required sample size that achieve a power of 0.80 [21].

\section{Instrumentation}

Grayson-Stadler (GSI) 61 Clinical Audiometer with ER-3A insert transducer and GSI TympStar Middle Ear Analyzer used to assess hearing thresholds and middle-ear function. Intelligent Hearing Systems Smart EP system used to record AMLR waveforms while subjects lying comfortably with eyes closed on a recliner chair using a rarefaction acoustic click stimuli of 100 microseconds duration and $75 \mathrm{~dB} \mathrm{nHL}$ at a rate of 7.1/second presented through gold foil tiptrode electrode placed in the right ear. Silver disk electrodes were applied according to the International 10/20 System with placements at Cz-A1 (contralateral recording) and Cz-A2 (Ipsilateral recording) relative to the right ear (test ear). Inter-electrode impedance was maintained below 5k AMLR. Click stimuli were band-pass filtered from $10-1500 \mathrm{~Hz}$ over a $56 \mathrm{~ms}$ time-base and averaged of 1000 sweeps. Two replications of each waveform were obtained for the pre-silence and post-silence conditions. The choice of right ear stimulation was based on the results of previous studies that showed no ear effect on the $\mathrm{Na}$, Pa components of AMLR measurements [20,22,23]. All measurements were obtained inside a double-walled test booth with ambient noise levels within the accepted specifications of the ANSI S3.1 1999 (R 2013) standards. Ear level measurements in dB SPL were $27.6 \mathrm{~dB}$ at $125 \mathrm{~Hz}$, $9.2 \mathrm{~dB}$ at $250 \mathrm{~Hz}, 4.8 \mathrm{~dB}$ at $500 \mathrm{~Hz}, 7.5 \mathrm{~dB}$ at $1000 \mathrm{~Hz}, 10 \mathrm{~dB}$ at 2000 $\mathrm{Hz}, 8.4 \mathrm{~dB}$ at $4000 \mathrm{~Hz}$, and $9 \mathrm{~dB}$ at $8000 \mathrm{~Hz}$.

\section{Procedures}

Study Participants were prepared for the AMLR evaluation and given instructions for the silence experience as follows "You will sit in silence in this room for ten minutes. During this time, you need to be relaxed and awake. At the end of the experiment, you will complete a short questionnaire about the silence period". Participants' attention was not directed toward auditory perceptions, and during instructions the word "tinnitus" was not used, so the participants' perceptions and responses would not be biased.

Baseline AMLR recordings were obtained first, then the participants sat in silence for ten minutes followed by a second AMLR re- cordings. A silence questionnaire was then administered to document any possible sound perception during silence experience (see Appendix A).

\section{Data analysis}

AMLR $\mathrm{Na} / \mathrm{Pa}$ relative amplitude $(\mu \mathrm{V})$ and absolute latencies (ms) for ipsilateral and contralateral recordings in both pre- and post-silence conditions were analyzed using repeated-measures ANOVA to examine the effect of silence and tinnitus perception. Wave $\mathrm{V}$ of the auditory brainstem response was present in the recordings and was within the normal range for all subjects, suggestive of normal function in the neural auditory pathway preceding the AMLR.

\section{Results}

A summary of the sample characteristics for participants who perceived tinnitus during silence exposure (tinnitus-perceiving group) and those who did not perceive tinnitus (non-tinnitus perceiving group) is displayed in table 1 . No statistically significant difference observed between groups with regard age 0 or the mean pure-tone averages (PTA) for either right (RE) or left ears (LE) (; LE: ). The group mean audiometric thresholds for right and left ears are illustrated in figures 1 and 2, respectively.

\begin{tabular}{|c|c|c|c|}
\hline Characteristic & $\begin{array}{c}\text { Tinnitus- } \\
\text { perceiving } \\
\text { group }\end{array}$ & $\begin{array}{c}\text { Non-tinnitus } \\
\text { perceiving } \\
\text { group }\end{array}$ \\
\hline Age & M (SD) & $23.82(6.19)$ & $24.67(1.08)$ \\
\hline PTA/right ear & M (SD) & $6.09(2.35)$ & $6.33(2.95)$ \\
\hline PTA/left ear & M (SD) & $6.54(2.75)$ & $6.17(3.14)$ \\
\hline Tinnitus perception & $\mathrm{N}(\%)$ & $33(55 \%)$ & $27(45 \%)$ \\
\hline $\begin{array}{c}\text { M= Mean, SD= } \\
\text { Standard Deviation, } \\
\text { N= Number of par- } \\
\text { ticipants, PTA= Pure } \\
\text { Tone Average. }\end{array}$ & & & \\
\hline
\end{tabular}

Table 1: Summary Characteristics.

\section{Tinnitus Perception during silence}

Overall, $55 \%$ of the participants in the current study perceived tinnitus during silence. All tinnitus subjects reported these perceptions within the first five minutes of silence with the majority re- 


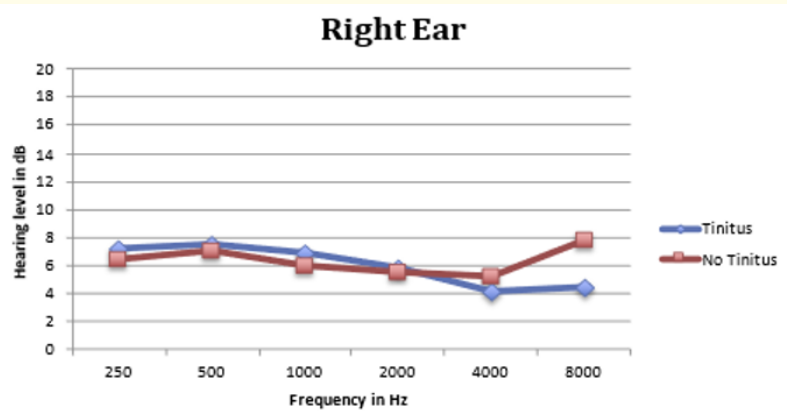

Figure 1: Right Ear Mean Audiograms Threshold for Tinnitus and Non-Tinnitus perceiving Groups.

porting more than one sound during silence. Table 2 displays the frequency and percentage of tinnitus onset and number of tinnitus sounds perceived during silence. Different tinnitus sounds per-

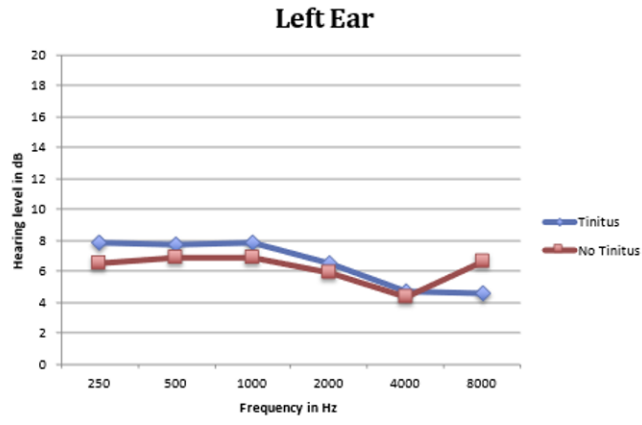

Figure 2: Left Ear Mean Audiograms Threshold for Tinnitus and Non-Tinnitus perceiving Groups.

ceived by the participants during silence are displayed in table 3 . The most commonly hear tinnitus sound was humming and buzzing.

\begin{tabular}{|c|c|c|c|c|c|}
\hline T. Onset & Frequency & Percent & Number of auditory perceptions & Frequency & Percent \\
\hline Immediately & 3 & $9.1 \%$ & 1 & 12 & $36.4 \%$ \\
\hline Within 2 min. & 13 & $39.4 \%$ & 2 & 12 & $36.4 \%$ \\
\hline Within 5 min. & 17 & $51.5 \%$ & 3 & 7 & $21.2 \%$ \\
\hline At 10 min. & 0 & $0 \%$ & 5 & 1 & $3 \%$ \\
\hline & & & Total & 33 & $100 \%$ \\
\hline Total Tinnitus & 33 & $100 \%$ & & $3 \%$ & 7 \\
\hline
\end{tabular}

Table 2: Tinnitus Onset and number of tinnitus sounds perceived during silence.

\begin{tabular}{|c|c|c|c|}
\hline Type & Sound & Number & Percent \\
\hline \multirow{4}{*}{ Non-Pulsatile } & Other & 13 & $39 \%$ \\
\cline { 2 - 4 } & Hum & 10 & $30 \%$ \\
\cline { 2 - 4 } & Buzz & 9 & $27 \%$ \\
\cline { 2 - 4 } & Roar & 4 & $12 \%$ \\
\cline { 2 - 4 } & Crickets & 2 & $6 \%$ \\
\cline { 2 - 4 } & Ringing & 1 & $3 \%$ \\
\cline { 2 - 4 } & Hiss & 1 & $3 \%$ \\
\cline { 2 - 4 } & Whistling & 1 & $3 \%$ \\
\cline { 2 - 4 } & Whizzing & 1 & $3 \%$ \\
\cline { 2 - 4 } & Running water & 0 & $39 \%$ \\
\hline \multirow{4}{*}{ Pulsatile } & Pulse & 13 & $36 \%$ \\
\hline
\end{tabular}

Table 3: Types of Tinnitus Sounds Heard by Subjects During Silence. 


\section{AMLR neural activity and tinnitus perception}

Grand average ipsilateral AMLR waveforms for tinnitus and non-tinnitus perceiving groups for both pre-silence and post-silence recordings are displayed in figure 3. Mean values for AMLR $\mathrm{Na}$, Pa latencies and relative amplitudes for ipsilateral and contralateral recordings are displayed in tables 4 and 5, respectively. Repeated-measures ANOVA showed no statistically significant group difference as a result of tinnitus perception on ipsilateral AMLR Na latency $(=0.005, \rho=0.95)$, Pa latency $(0.74, \rho=0.39)$ or contralateral AMLR Na latency $(=1.81, \rho=0.19)$, Pa latency $(=1.3, \rho=0.26)$, $\mathrm{Na} / \mathrm{Pa}$ amplitudes $(=3.82, \rho=0.056)$. However, AMLR contralateral $\mathrm{Na} / \mathrm{Pa}$ amplitude were larger in tinnitus subjects, the difference was not statistically significant. The results of the repeated-measures ANOVA did reveal a statistically significant difference in the ipsilateral $\mathrm{Na} / \mathrm{Pa}$ wave amplitude (7.396, $\rho=0.009$ ), with the $\mathrm{Na}$ / $\mathrm{Pa}$ amplitudes being larger in tinnitus perceiving subjects in both pre-silence and post-silence recordings. These results indicate that participants who perceived tinnitus had a larger AMLR activity at baseline (before and after silence).

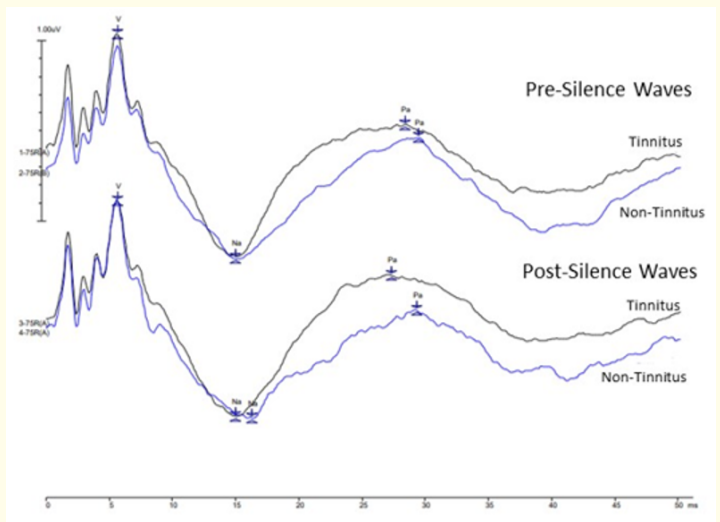

Figure 3: Grand average AMLR waveform recorded from tinnitus and non-tinnitus perceiving groups.

\begin{tabular}{|c|c|c|c|c|}
\hline \multirow{2}{*}{ Parameter } & \multicolumn{2}{|c|}{ Pre-silence } & \multicolumn{2}{c|}{ Post-silence } \\
\cline { 2 - 5 } & Tinnitus & No Tinnitus & Tinnitus & No Tinnitus \\
\cline { 2 - 5 } & M (SD) & M (SD) & M (SD) & M (SD) \\
\hline Wave Na Latency (ms) & $16.16(1.72)$ & $15.93(1.67)$ & $15.71(1.57)$ & $15.99(1.74)$ \\
\hline Wave Pa Latency (ms) & $28.51(3.22)$ & $29.16(3.48)$ & $27.9(3.0)$ & $28.6(3.5)$ \\
\hline Wave Na/Pa Ampl $((\mu \mathrm{V})$ & $1.2(0.54)$ & $0.91(0.34)$ & $1.2(0.57)$ & $0.92(0.23)$ \\
\hline \multicolumn{7}{|c|}{ M= Mean, SD= Standard Deviation; Ampl= Amplitude. } \\
\hline
\end{tabular}

Table 4: Ipsilateral AMLR Measures for pre-silence and post-silence recordings.

\begin{tabular}{|c|c|c|c|c|}
\hline \multirow{2}{*}{ Parameter } & \multicolumn{2}{|c|}{ Pre-silence } & \multicolumn{2}{c|}{ Post-silence } \\
\cline { 2 - 5 } & Tinnitus & No Tinnitus & Tinnitus & No Tinnitus \\
\cline { 2 - 5 } & M (SD) & M (SD) & M (SD) & M (SD) \\
\hline Wave Na Latency $(m s)$ & $16.6(1.9)$ & $16.3(1.5)$ & $16.2(1.8)$ & $16.5(1.5)$ \\
\hline Wave Pa Latency $(m s)$ & $29.02(3.47)$ & $29.86(3.77)$ & $28.48(3.3)$ & $29.5(3.44)$ \\
\hline Wave Na/Pa Ampl $((\mu \mathrm{V})$ & $1.2(0.84)$ & $0.91(0.39)$ & $1.3(0.36)$ & $1.1(0.9)$ \\
\hline
\end{tabular}

Table 5: Contralateral AMLR Measures for pre-silence and post-silence recordings

\section{Effect of silence on AMLR measurements}

No statistically significant effect of silence on ipsilateral AMLR Na latency ( $=0.84, \rho=0.36)$, Pa latency $(=3.91, \rho=0.053)$; or $\mathrm{Na}$ /
Pa amplitude ( $=0.02, \rho=0.97)$. There was no interaction effect of silence and tinnitus for Na latency $(=1.53, \rho=0.22)$, Pa latency $(=0.002, \rho=0.97)$, nor $\mathrm{Na} / \mathrm{Pa}$ amplitude $(=0.001, \rho=0.98$ ) for 
ipsilateral measurement. Similarly, results revealed no significant effect of silence on contralateral AMLR measures Na latency $(=$ $0.13, \rho=0.72$ ), Pa latency ( $=1.31, \rho=0.26)$, or $\mathrm{Na} / \mathrm{Pa}$ amplitude ( $=0.13, \rho=0.72$ ). There was no interaction effect of silence and tinnitus for Na latency ( $=1.81, \rho=0.18$ ), Pa latency ( $=0.05, \rho=0.18$ ), nor $\mathrm{Na} / \mathrm{Pa}$ amplitude ( $=1.8, \rho=0.19$ ). These results indicate no change over time for AMLR waveforms in both groups compared to initial measurements. Estimated marginal means of ipsilateral and contralateral $\mathrm{Na} / \mathrm{Pa}$ Amplitude for tinnitus and non- tinnitus perceiving groups in pre-silence and post-silence conditions displayed in figures 3 and 5 , respectively.

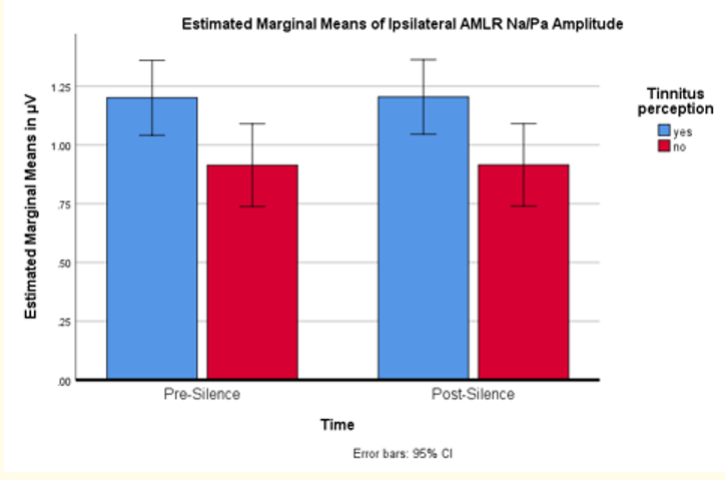

Figure 4: Estimated Marginal Means of Ipsilateral $\mathrm{Na} / \mathrm{Pa}$ Amplitude for Tinnitus and Non-Tinnitus perceiving Groups.

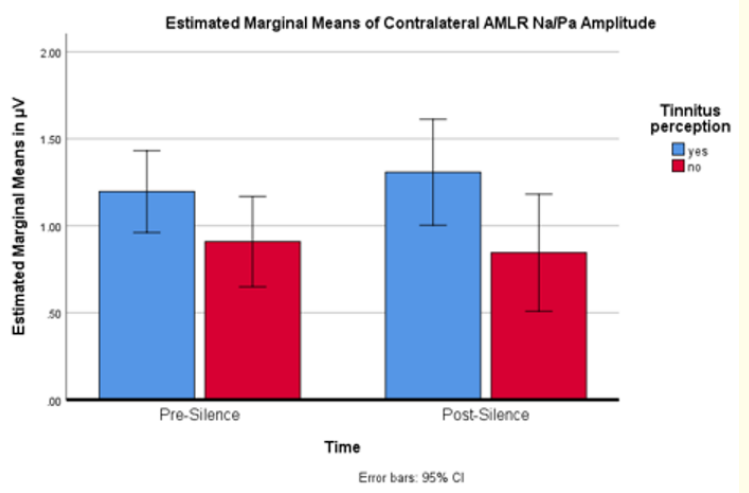

Figure 5: Estimated Marginal Means of Contralateral $\mathrm{Na} / \mathrm{Pa} \mathrm{Am}-$ plitude for Tinnitus and Non-Tinnitus perceiving Groups.

\section{Discussion}

Results demonstrated that a brief period of silence in a sound booth is sufficient to induce tinnitus perceptions in healthy normal hearing female subjects without directed auditory attention. $55 \%$ of study participants perceived various tinnitus sounds in the first five minutes of silence with no observed difference in hearing thresholds between tinnitus perceiving and non-tinnitus perceiving groups. This finding was substantially lower than that reported by Heller and Bergman [17] (94\%) and Del Bo., et al. [16] (83\%). Results are comparable to those of Tucker., et al. [19] (64\%) and Knobel and Sanchez [18] who reported $68.2 \%$ of their cohort perceived tinnitus during silence with directed auditory attention, $45.5 \%$ perceived tinnitus during silence with directed visual attention, and $19.7 \%$ perceived tinnitus during silence while engaged in a cognitive task. One reason for the higher percentage of tinnitus perception in the Heller and Bergman study [17] was the selfreport of hearing ability. Undetected hearing loss might lead to a higher tinnitus perception in their participants. Another reason for the higher percentage of tinnitus in the previous studies is the level of directed auditory attention to tinnitus perceptions in which topdown influence of attention and expectation might have modulated neural responses. In previous studies, attention was directed to the auditory system by instructing the participants at the beginning of the silence experiment to note any sounds that they might perceive during silence. In contrast, the present study focused on directing attention away from sound perception by instructing subjects that the purpose of the study is to examine the effect of silence on the auditory system. Differences among findings of previous studies and the present study may be related to the age range for the participants. In this study participants' ages ranged from 18-40, while participants in previous studies had a broader age range For example, Knobel and Sanchez [16] participant's ages ranged from 18 to 65 years.

All participants in the present study had normal hearing thresholds better than $10 \mathrm{~dB}$ between $250-8000 \mathrm{~Hz}$ (clinically normal limits), which does not exclude cochlear pathologies at a higher frequency range [24]. Although the results of the current study showed no difference regarding the clinical hearing threshold between tinnitus and non-tinnitus subjects, one cannot discount the possibility of hidden cochlear pathology that might have contributed to tinnitus perception during silence. However, the results of 
Del Bo., et al. [16] revealed a high prevalence of temporary tinnitus perception during silence while using more restrictive inclusive criteria that included thresholds up to $16000 \mathrm{~Hz}$, tympanometry, and distortion product otoacoustic emissions DPOAEs. In the present study, the most common tinnitus perceptions during silence other than pulse and heartbeat are hum and buzz. This finding is similar to those of Del Bo., et al. [16] and relatively similar to those of Heller and Bergman [17], and Tucker., et al. [19] who reported "ringing" and "Buzz" to be the most common tinnitus perception during silence.

The most significant finding in the present study was that the underlying neural activity that contributes to AMLR recordings was significantly larger in the tinnitus-perceiving group compared non-tinnitus perceiving group. The difference in this activity was not provoked by the effect of silence on the auditory system. Instead, the difference in AMLR waveform activity was already present at baseline testing. These results suggest the presence of higher spontaneous central neurophysiological activities in tinnitus perceiving subjects and that auditory deprivation may facilitate the perception of tinnitus perception in the absence of masking environmental sounds.

Several participant factors can affect AMLR waveforms amplitude and latency, such as age, gender, and the presence of hearing loss [25]. Age can impact AMLR measurements with the possibility of age-related decline in the inhibitory circuit within the auditory system that is mediated by gamma-aminobutyric acid causing larger AMLR amplitudes above the age of sixty years [26]. However, in the current study, there was no statistically significant difference in age or hearing thresholds among participants in the tinnitusperceiving and non-tinnitus perceiving groups. Gender can also impact AMLR measures as Tucker., et al. [27] demonstrating longer $\mathrm{Pa}$ latencies in male subjects and larger $\mathrm{Pa}$ amplitudes in female subjects were reported. As all participants in the current study were female, differences in AMLR waveform amplitudes were not related to gender.

Several putative mechanisms might have contributed to these results, First, unmasked spontaneous activity of the auditory nerve and higher auditory centers through a loss of lateral inhibition may lead to tinnitus perceptions during silence [28,29]. In the present study, larger AMLR amplitude was observed in participants in both pre-silence and post-silence recordings in tinnitus-perceiving sub- jects, which indicates an underlying difference in spontaneous neural activity between groups. This mechanism highlights the impact of the surrounding acoustical environment on tinnitus perception. Many subjects with chronic tinnitus report that their tinnitus perception is more prominent in quiet settings and less perceived in sound enriched environments. This phenomenon is supported by the role of sound therapy in tinnitus management [13]. Jastreboff [13] has proposed that tinnitus signals may exist in individuals' networks at low strength and can be perceived only when the surrounding background sounds are low. Animal studies reveal that reorganization of the tonotopic map of the auditory cortex as well as increased spontaneous firing rate and neural synchrony are more pronounced in quiet environments compared with sound enriched environments [30]. This mechanism denotes that tinnitus perception during silence may represent higher ongoing activities within the auditory system in tinnitus-perceiving subjects and that surrounding environmental sounds play an important role in mitigating the perception of these activities.

A second putative mechanism for tinnitus perception during silence maybe gain modulation in the CANS in response to reduced sensory inputs to preserve a stable neural coding efficiency [31]. This modulation may result in an overall increased sensitivity and tinnitus perceptions. While the resulting over-amplification of spontaneous activity in this scenario could account for emerging tinnitus perception, silence should influence AMLR amplitude in which post-silence recordings would have a larger amplitude compared to pre-silence recording in both groups. However, the present study found that silence did not a significant effect on AMLR neural activity in both groups. Increased underlying AMLR neural activity in tinnitus perceiving subjects was observed in both presilence and post-silence recordings, suggestive of an initial difference between groups in neurophysiological activities before silence exposure. These results indicate that silence was a facilitating factor for tinnitus perception but not the triggering one for these differences in neural activities between groups.

\section{Conclusion}

In conclusion, tinnitus perception may occur in nonclinical population when exposed to a brief period of silence. Substantial concomitant higher central neurophysiological activities were associated with these tinnitus perceptions as reflected in larger AMLR waveform amplitude in both pre-silence and post-silence record- 
ings in tinnitus-perceiving subjects. Additional research is needed to examine CANS activities associated with tinnitus perception during silence and to explore different personality traits that can impact these perceptions.

\section{Conflict of Interest}

No financial interest or any conflict of interest exists.

\section{Bibliography}

1. Møller AR. "Sensorineural Tinnitus: Its Pathology and Probable Therapies". International Journal of Otolaryngology 2016 (2016): 2830157.

2. Baguley D., et al. "Tinnitus". The Lancet 382.9904 (2013): 1600-1607.

3. Langguth B., et al. "Tinnitus and depression". The World Journal of Biological Psychiatry 12.7 (2011): 489-500.

4. Møller AR. "Tinnitus: presence and future”. In: Langguth B, Hajak G, Kleinjung T, Cacace A, Møller AR, eds. Progress in Brain Research". Tinnitus: Pathophysiology and Treatment. Elsevier 166 (2007): 3-16.

5. Møller AR. "Sensorineural Tinnitus: Its Pathology and Probable Therapies". International Journal of Otolaryngology.

6. Eggermont JJ and Roberts LE. "The neuroscience of tinnitus". Trends in Neuroscience 27.11 (2004): 676-682.

7. Georgiewa P., et al. "An integrative model of developing tinnitus based on recent neurobiological findings". Medical Hypotheses 66.3 (2006): 592-600.

8. Nemati S., et al. "Cochlear and brainstem audiologic findings in normal hearing tinnitus subjects in comparison with nontinnitus control group". Acta Medica Iranica 52.11 (2014): 822-826.

9. Saunders JC. "The role of central nervous system plasticity in tinnitus". Journal of Communication Disorders 40.4 (2007): 313-334.

10. Møller AR., et al. "Textbook of Tinnitus". Springer-Verlag (2011). doi:10.1007/978-1-60761-145-5

11. Schaette R., et al. "Reversible induction of phantom auditory sensations through simulated unilateral hearing loss". PLoS One 7.6 (2012): e35238.
12. Hoare DJ., et al. "Sound therapy for tinnitus management: practicable options". Journal of the American Academy of Audiology 25.1 (2014): 62-75.

13. Jastreboff MM. "Sound therapies for tinnitus management”. In: Langguth B, Hajak G, Kleinjung T Cacace A. Progress in Brain Research. Tinnitus: Pathophysiology and Treatment. Elsevier 166 (2007): 435-440.

14. Tyler RS., et al. "Tinnitus Sound Therapy Trial Shows Effectiveness for Those with Tinnitus". Journal of the American Academy of Audiology 31.1 (2020): 6-16.

15. King ROC., et al. "The Effect of Auditory Residual Inhibition on Tinnitus and the Electroencephalogram". Ear and Hearing 42.1 (2021): 130-141.

16. Del Bo L., et al. "Tinnitus aurium in persons with normal hearing: 55 years later". Otolaryngology-Head and Neck Surgery 139.3 (2008): 391-394.

17. Heller MF and Bergman M." Tinnitus aurium in normally hearing persons". The Annals of Otology, Rhinology, and Laryngology 62.1 (1953): 73-83.

18. Knobel KAB and Sanchez TG. "Influence of silence and attention on tinnitus perception". Otolaryngology-Head and Neck Surgery 138.1 (2008): 18-22.

19. Tucker DA., et al. "The effect of silence on tinnitus perception". Otolaryngology-Head and Neck Surgery 132.1 (2005): 20-24.

20. Hall JW. "New Handbook of Auditory Evoked Responses". Pearson (2007).

21. Cohen J. "A power primer". Psychological Bulletin 112.1 (1992): 155-159.

22. Tucker DA., et al. "Effect of stimulus intensity level on auditory middle latency response brain maps in human adults". Journal of the American Academy of Audiology 12.5 (2001): 223-232.

23. Weihing J and Musiek F. "The influence of aging on interaural asymmetries in middle latency response amplitude". Journal of the American Academy of Audiology 25.4 (2014): 324-334.

24. Buzo BC and Carvallo RMM. "Psychoacoustic analyses of cochlear mechanisms in tinnitus patients with normal auditory thresholds". International Journal of Audiology 53.1 (2014): 40-47. 
25. Theodoroff S., et al. "Auditory middle latency responses in individuals with debilitating tinnitus". The International Tinnitus Journal 16.2 (2011): 104-110.

26. Picton TW. "Human Auditory Evoked Potentials". Plural Pub (2011).

27. Tucker DA., et al. "Effects of stimulus rate and gender on the auditory middle latency response". Journal of the American Academy of Audiology 13.3 (2002): 146-153.

28. Kral A and Majernik V. "On lateral inhibition in the auditory system". General Physiology and Biophysics 15.2 (1996): 109-

127.

29. Salvi RJ., et al. "Auditory plasticity and hyperactivity following cochlear damage". Hearing Research 147.1 (2000): 261-274.

30. Noreña AJ and Eggermont JJ. "Enriched acoustic environment after noise trauma abolishes neural signs of tinnitus". Neuroreport 17.6 (2006): 559-563.

31. Noreña AJ. "An integrative model of tinnitus based on a central gain controlling neural sensitivity". Neuroscience and Biobehavioural Reviews 35.5 (2011): 1089-1109.

Volume 3 Issue 12 December 2021

(c) All rights are reserved by Marwa F Abdrabbou and Denise A Tucker. 